\title{
New recurrent BRCA1/2 mutations in Polish patients with familial breast/ovarian cancer detected by next generation sequencing
}

\author{
Anna Kluska ${ }^{1}$, Aneta Balabas' ${ }^{1}$ Agnieszka Paziewska², Maria Kulecka², Dorota Nowakowska', Michal Mikula ${ }^{1 *}$ \\ and Jerzy Ostrowski ${ }^{1,2^{*}}$
}

\begin{abstract}
Background: Targeted PCR-based genetic testing for BRCA1 and BRCA2 can be performed at a lower cost than full gene testing; however, it may overlook mutations responsible for familial breast and/or ovarian cancers. In the present study, we report the utility of next generation sequencing (NGS) to identify new pathogenic variants of BRCA1/2.

Methods: BRCA1 and BRCA2 exons were amplified using the lon AmpliSeq BRCA1/2 Panel and sequenced on the lon Torrent PGM sequencer in 512 women with familial and/or only early onset breast and/or ovarian cancers who were negative for selected BRCA1/2 mutations.

Results: 146 single nucleotide variants (SNVs) and 32 indels were identified. Of them, 14 SNVs and 17 indels were considered as pathogenic or likely pathogenic. One and 18 pathogenic mutations had been detected previously in the Polish and other populations, respectively, and 12 deleterious mutations were previously unknown. Eight mutations were recurrent; Q563X (BRCA1), N3124I (BRCA2) and c.4516delG (BRCA1) were found in eight, six and four patients, respectively, and two other mutations (c.9118-2A > G and c.7249delCA in BRCA2) were detected in three patients each. Altogether, BRCA1/2 pathogenic mutations were identified in 52 out of 512 (10\%) patients.

Conclusions: NGS substantially improved the detection rates of a wide spectrum of mutations in Polish patients with familial breast and/or ovarian cancer. Although targeted screening for specific BRCA1 mutations can be offered to all Polish breast or ovarian cancer patients, NGS-based testing is justified in patients with breast or ovarian cancer likely related to $B R C A 1 / 2$ who test negative for the selected BRCA1/2 pathogenic mutations.
\end{abstract}

\section{Background}

Approximately $5-10 \%$ of breast cancers and $10 \%$ of ovarian cancers have a hereditary component [1], which in most cases is associated with clinically significant mutations in the BRCA1 and BRCA2 genes. According to the Breast Cancer Information Core (BIC) [2], more than 1800 and 2000 distinct variants of the BRCA1 and BRCA2 genes have been described, respectively. The incidence and spectrum of mutations vary among populations, and the carrier frequency in general populations ranges between $1 / 40$ and $1 / 800$ [3]. Some populations demonstrate a wide spectrum of mutations, while others are characterized by high prevalence of a small number of founder

\footnotetext{
* Correspondence: mikula.michal@gmail.com; jostrow@warman.com.pl 'Department of Genetics, Maria Sklodowska-Curie Memorial Cancer Center and Institute of Oncology, 02-781 Warsaw, Poland

Full list of author information is available at the end of the article
}

mutations [4]. In Ashkenazi Jews, three founder mutations in $B R C A 1$ and $B R C A 2$ comprise $80 \%$ to $95 \%$ of mutations, respectively [5,6]. In Poland, the frequency of $B R C A 1$ mutations is estimated to be between $1 / 240$ and $1 / 360$, and two founder mutations, namely, 5382insC and T300G (C61G), account for $70-90 \%$ of BRCA1 mutations [3]. None of them is unique to Poland.

Breast cancer is the most common cancer among Polish women, and is a leading cause of cancer-related morbidity and mortality [7]. The only factor that significantly decreases cancer-related mortality is early diagnosis, and in special cases, prophylactic surgery. Since cancers detected at an early stage of development are asymptomatic or low-symptomatic disorders, early diagnosis of cancer is usually accidental or the result of screening programs. Current cancer screening guidelines for the general population are based on the age of the individual, and patients 
at risk of developing cancer are identified on the basis of a positive family history of cancer. Families with two firstor second-degree relatives with breast and/or ovarian cancers, with at least one of the cases diagnosed before the age of 50 years, are considered to be at moderate risk, while those with at least three relatives with breast and/or ovarian cancers are considered to be at high risk [7].

The mutation status may determine the medical management of patients, including annual screening and prophylactic surgery, and genetic testing for $B R C A 1$ and $B R C A 2$ is routinely performed in women with hereditary breast and ovarian cancer risk. More than 100,000 individuals in the United States undergo BRCA testing annually [8]. However, since BRCA testing is a costly procedure (the current charge for full gene sequencing is several hundred to thousands of dollars), most genetic counseling programs recommend less expensive targeted screening for specific $B R C A 1$ and $B R C A 2$ mutations rather than full gene testing [8].

Next generation sequencing (NGS) offers a powerful, faster, and cheaper alternative for targeted sequencing aimed at identifying non-synonymous, splicing, and stop codon variants exhibiting deleterious consequences in the coding genes [9]. As a consequence, several NGS-based workflows for clinical $B R C A 1$ and $B R C A 2$ mutation testing were recently proposed [10-16].

In a previous study from our group, we used multiplexed PCR-based genotyping technology and showed that the frequency of selected BRCA1 mutations was moderate in familial and high in nonfamilial breast cancers. In addition, a strong founder effect was confirmed for $B R C A 1$ mutations but not for BRCA2 mutations in the Polish population [7]. In the present study, we show that the use of the Ion Torrent PGM sequencer to sequence the coding exons of BRCA1 and BRCA2 genes substantially improves the detection rates of a wide spectrum of mutations in Polish patients with familial and/or only early onset of breast and/or ovarian cancers.

\section{Methods}

\section{Patients}

Patients whose blood samples were collected between 2003 and 2010 were selected from the registry of the Genetic Counseling Unit, Warsaw Cancer Center-Institute of Oncology. The personal and familial cancer histories were acquired by in-depth interviews. Healthy individuals with no known personal or familial history of malignancy, normal results of screening colonoscopy, and normal mammography or PSA levels were recruited primarily from the National Colorectal Cancer Screening Program. All patients and control subjects were Polish Caucasians recruited from the Masovian voivodeship population. Informed consent for hereditary cancer genetic testing was obtained from all of the patients. The permission for genetic testing was granted by the Ethical Committee at Maria Sklodowska-Curie Memorial Cancer Center and Institute of Oncology on 9 May 2002 (No. 28/2002) and further extended to include testing with NGS on 11 June 2013 (No. 28/2002/1/2013). The study did not require ethical approval.

\section{BRCA $1 / 2$ sequencing}

Genomic DNA was extracted from whole blood as described previously [7]. DNA concentration was determined on a Qubit 2.0 Fluorometer using the Qubit dsDNA BR Assay Kit (Life Technologies). For library preparation, a set of reagents from the Ion AmpliSeq ${ }^{\mathrm{Tw}} \mathrm{Li}$ brary Kit 2.0 (Life Technologies) and Ion AmpliSeq BRCA1 and BRCA2 Panel, which comprises 167 primer pairs spanning $16.25 \mathrm{~kb}$, were used to amplify the coding regions of the BRCA1 and BRCA2 genes. Amplification for each patient was performed using three separate primer pools containing $1.4 \mu \mathrm{l}$ of $5 \times$ Ion AmpliSeq $^{\text {Tm }} \mathrm{HiFi}$ Master Mix, $3.5 \mu \mathrm{l}$ of $2 \times$ Ion AmpliSeq Primer Pool, and $3 \mathrm{ng}$ of DNA in a total reaction volume of $7 \mu$ l. Cycling conditions were as follows: $99^{\circ} \mathrm{C}$ for $2 \mathrm{~min}$, followed by 19 cycles of $99^{\circ} \mathrm{C}$ for $15 \mathrm{sec}$ and $60^{\circ} \mathrm{C}$ for $4 \mathrm{~min}$, ending with a hold at $10^{\circ} \mathrm{C}$. At this point, three separate reactions for a given patient were combined, and amplicons were partially digested at primer sequences with $2 \mu \mathrm{l}$ of FuPa reagent under conditions provided by the manufacturer in The Ion AmpliSeq DNA and RNA Library Preparation protocol (Revision B.0). Subsequent steps of library preparation were performed according to the above protocol. Briefly, following digestion, samples were subjected to sequencing adapter ligation with either 16 or 32 indexing barcodes, and purified with the AMPure XP reagent (Beckman Coulter). The DNA library was eluted from AMPure XP beads with $52 \mu \mathrm{l}$ of PCR reagent solution consisting of $50 \mu \mathrm{l}$ of Platinum ${ }^{\circ}$ PCR Super Mix HiFi and $2 \mu \mathrm{l}$ of Library Amplification Primer Mix, and amplified under the following conditions: $98^{\circ} \mathrm{C}$ for $2 \mathrm{~min}$, followed by five cycles of $98^{\circ} \mathrm{C}$ for $15 \mathrm{sec}$ and $64^{\circ} \mathrm{C}$ for $1 \mathrm{~min}$, ending with a hold at $10^{\circ} \mathrm{C}$. Next, the amplified DNA library was subjected to a two-round purification process using AMPure XP beads with $0.5 \times$ and $1.2 \times$ bead-to-sample volume ratios, respectively. Library concentrations were determined with the Qubit dsDNA HS Assay Kit (Life Technologies), and the respective size distributions were determined with the High Sensitivity DNA Analysis Kit on Bioanalyzer 2100 (Agilent).

Library molarity was determined, and either 16 or 32 libraries were pooled in equimolar concentrations and used for automatic template preparation on Ion Chef using reagents from the Ion PGM IC 200 Kit and Ion 316 Chip Kit v2 BC. Sequencing was performed on the Ion Torrent PGM sequencer using 500-flow runs. Data from the PGM runs were processed on the Ion Torrent server using a 
platform-specific pipeline incorporated in Torrent Suite v4.2.1 (Life Technologies) to obtain sequence reads, trim adapter sequences, filter and remove poor signal reads, and assign the reads to a given barcode. The reads were mapped to the hg19 (Homo sapiens) reference genome and adjusted to the specific amplicon target regions deposited in the "BRCA1_2.20131001.designed" BED file available in Torrent Suite. The coverageAnalysis (v4.2.1.4) and variantCaller (v4.2.1.0) plug-ins with a set of default parameters optimized for the BRCA1/BRCA2 panel were obtained from www.Ampliseq.com, and were ran for each sequencing dataset.

\section{Post-sequencing variant analysis}

Variants were classified either as single nucleotide variants (SNVs) or insertion/deletion (indels) variants using a python script. Variants were annotated using data from BIC for the $B R C A 1$ and $B R C A 2$ genes. The identified variants were matched to existing variants in BIC according to four parameters as follows: chromosome number (chromosome 13 and chromosome 17 for BRCA2 and BRCA1, respectively); position of the variant in the human genome assembly hg19 (GRCh37), which was retrieved from the BIC database field HGVS; genomic and type of reference; and alternate base. The possibility of different alternate and reference base was taken into account as well as the possibility of variant on the reverse strand.

All the SNVs were analyzed using Variant Effect Predictor [17] (VEP) for the human genome assembly hg19 (GRCh37). The occurrence of variants was determined using an R script. Variants with unknown clinical significance in BIC and new changes were subjected to in silico analysis of their deleteriousness, and screened for evidence of pathogenicity in the literature.

\section{Selection of pathogenic variants}

SNVs and indels were analyzed using Condel [18] and SIFT Indel [19], respectively. Missense variants were classified as pathogenic when reported in BIC as pathogenic, and/or predicted as pathogenic by a Condel score, and identified through a literature search [20-29]. Frameshift mutations were classified as pathogenic when reported as pathogenic by BIC and/or predicted as pathogenic by SIFT Indel.

\section{Genotyping}

Pathogenic mutations detected by NGS were confirmed by Sanger sequencing. In addition, five selected variants, namely, Q356R, N3124I, Q563X, c.7249delCA, and c.9118-2G > A, were genotyped using Custom TaqMan SNP Genotyping Assays (Life Technologies, USA) and a 7900HT Real-Time PCR system (Life Technologies) as described previously [7].

\section{Results}

\section{NGS analysis}

The incidence of SNVs and indels within 167 amplicons covering the BRCA1 and BRCA2 exons was tested using the Ion Torrent PGM sequencer in 31 (a training set) and 512 women with breast cancer or ovarian cancer newly diagnosed before the age of 50 years who were positive and negative for $B R C A 1 / 2$ mutations, respectively, as determined by targeted genotyping. The genotyping comprised 11 mutations in BRCA1, namely c.66_67delAG, C61R, c.3700_3704del5, c.3756delGTCT, c.3777delT, c.4035delA, c.4041delAG, c.4065delTCAA, c.5263delC, $\mathrm{R} 1738 \mathrm{E}$ and R1751X, and nine mutations in BRCA2, namely E394X, c.5239insT, c.5946delT, c.5964delAT, c.6447delTA, c.7910del5, c.8924delT, R3128X and c.9402delT [7,30]. Of the 512 women, 317 had familial breast and/or ovarian cancer, and 195 had only early onset cancer and/or contralateral breast and ovarian cancers; the median age of women with breast cancer at diagnosis was 43 years.

The average number of bases with $\geq Q 17 / \geq Q 20$ across all 21 PGM sequencing runs was 343/324 Mbp, constituting $96 / 90 \%$ of total output. The average coverage across all samples was 507, whereas the mean mapping rate reached $94 \%$.

The NGS-based procedure confirmed the pathogenic mutations in all 31 DNA samples of a training set (not shown). Among 512 DNA samples analyzed, we identified 146 SNVs (64 in BRCA1 and 82 in BRCA2) and 32 indels (20 in BRCA1 and 12 in BRCA2). Sixteen indels were found in coding regions (nine in $B R C A 1$ and seven in $B R C A 2)$. The results of the detailed analysis of SNVs and indels are summarized in Additional file 1.

The types and number of SNVs are summarized in Table 1. Of these, eight SNVs (five in BRCA1 and three in $B R C A 2)$ were already reported in $\mathrm{BIC}$ as pathogenic. Three additional SNVs were reported as variants of uncertain significance (VUS); variants co-localized with these SNVs were reported as pathogenic in ClinVar. In addition, we identified three novel nonsense variants (Table 2). Of 41 missense variants that were either reported as VUS in BIC or had not been reported previously, 24 had at least one transcript in which the Condel score was reported as deleterious (Table 3).

Of the indels identified in the present study, five in $B R C A 1$ and two in BRCA2 had already been reported in $\mathrm{BIC}$ as pathogenic. One additional indel was reported as unknown in BIC, although its co-located variants in dbSNP were reported in ClinVar as pathogenic (Table 4). We also found nine novel indels in coding regions (Table 4).

Altogether, among 512 patients in whom the previous targeted genotyping did not reveal a pathogenic BRCA1/2 mutation massive amplicon sequencing identified 52 patients 
Table 1 Types and number of single nucleotide variants in the BRCA1 and BRCA2 genes

\begin{tabular}{lll}
\hline Type of variant & Number & \\
\cline { 2 - 3 } & BRCA1 & BRCA2 \\
\hline Intronic variant & 21 & 23 \\
Intronic variant near splice site & 3 & 4 \\
Missense variant & 25 & 36 \\
Nonsense variant & 6 & 3 \\
Synonymous variant & 4 & 14 \\
Variant in the splice region of exon & 0 & 1 \\
3' or 5' UTR variants & 5 & 1 \\
\hline
\end{tabular}

with 31 deleterious mutations, of which sixteen were frameshift, eight were nonsense, three were missense, and four affected splicing. Of note, one patient carried two pathogenic mutations, namely Q563X and N3124I. All pathogenic variants were confirmed by Sanger direct sequencing. Five other variants were likely pathogenic, and 17 variants were VUS. All of them were missense mutations (Table 3).

\section{Genotyping for NGS-selected mutations}

Using the TaqMan SNP Genotyping Assay, the incidence of five selected variants, one common polymorphism (Q356R) and four mutations (N3124I, Q563X, c.7249delCA, and c.9118-2G > A) were tested in additional group of 445 cancer patients diagnosed under the age of 50 and 1300

Table 2 Pathogenic single nucleotide variants in BIC and ClinVar

\begin{tabular}{|c|c|c|c|}
\hline Variant & Type & Evidence of pathogenicity & Count \\
\hline \multicolumn{4}{|l|}{ BRCA1 } \\
\hline Q563X* & Nonsense & BIC, ClinVar & 8 \\
\hline W1782X & Nonsense & BIC, ClinVar & 2 \\
\hline Y1563X & Nonsense & BIC, ClinVar & 1 \\
\hline Q538X & Nonsense & BIC, ClinVar & 1 \\
\hline E1415X & Nonsense & & 1 \\
\hline C1501X & Nonsense & & 1 \\
\hline R1699W & Missense & BIC, ClinVar & 2 \\
\hline \multicolumn{4}{|l|}{ BRCA2 } \\
\hline Q92X & Nonsense & BIC, ClinVar & 1 \\
\hline$c .9118-2 A>G$ & IVS & BIC, ClinVar & 3 \\
\hline P3039P & Splice & BIC, ClinVar & 1 \\
\hline Q3047X & Nonsense & & 1 \\
\hline N3124l & Missense & ClinVar, Condel & 6 \\
\hline $12627 F$ & Missense & ClinVar, Condel & 1 \\
\hline$c .8754+5 G>T$ & IVS & ClinVar & 2 \\
\hline
\end{tabular}

* Patients with ovarian and breast cancer.

IVS intronic variant near splice site; Splice variant in the splice region of exon. healthy individuals (856 women and 444 men with a median age of 59 years).

Although Q356R was described as pathogenic in ClinVar, its prevalence was similar among patients (15.1\%) and healthy controls (16.9\%). Therefore, the clinical significance of this polymorphic variant was not confirmed. By contrast, Q563X was detected in 5 (1.1\%) out of 445 cancer patients, respectively, but only in one healthy individual. The three other mutations (N3124I, c.7249delCA, and c.9118-2G > A) were detected by TaqMan genotyping only in DNA samples in which they were identified by NGS.

\section{Discussion}

The rare germline pathogenic $B R C A 1$ mutations increase the risk of breast and ovarian cancer to approximately $80 \%$ and $60 \%$, respectively, whereas BRCA2 mutations increase breast cancer risk to more than $80 \%$ by the age of 80 [10]. Because the risk of cancer can be predicted by a family history and/or by an early disease onset, the Genetic Counseling Unit at the Warsaw Cancer CenterInstitute of Oncology offers risk counseling based on the occurrence of cancer in the pedigree. However, according to Polish guidelines and recommendations, the government sponsored surveillance program, which includes annual mammography and breast magnetic resonance imaging, pelvic ultrasonography, and cancer antigen 125 levels, is provided only to women with known pathogenic mutations. Therefore, in families with an increased cancer risk, genetic testing is critical for preventive healthcare, including specialized surveillance programs and prophylactic mastectomy and/or oophorectomy, which reduce the risk of breast/ovarian cancer by over 90\% [31]. The detection of $B R C A 1 / 2$ pathogenic mutations facilitates the selection of patients and the delivery of healthcare and related services in women harboring their respective germline mutations.

Although BRCA1 and BRCA2 mutations [32-37] are present in more than $60 \%$ and $30 \%$ of families, respectively, our targeted mutation screening [7] detected 20.8\% and $1.3 \%$ of patients carrying pathogenic BRCA1 and $B R C A 2$ mutations, respectively. This suggests that a proportion of mutations responsible for familial breast and/or ovarian cancers were not identified. In the present study, we report the utility of NGS for the detection of new pathogenic variants of $B R C A 1 / 2$.

Among 512 DNA samples analyzed, we identified 146 SNVs and 32 indels, of which 14 SNVs and 17 indels were considered as pathogenic or likely pathogenic. One and 18 pathogenic mutations had been detected previously in the Polish and other populations, respectively, and 12 deleterious mutations had not been detected earlier. Eight mutations were recurrent; Q563X, N3124I and c.4516delG were found in eight, six and four patients, respectively, and two other mutations (c.9118-2A > G and c.7249delCA) were 
Table 3 Pathogenic missense variants according to the Condel algorithm

\begin{tabular}{|c|c|c|c|c|c|c|c|}
\hline Variant & Gene & rs & Count & Condel count & Condel max & Condel algorithms & Confirmed by other models \\
\hline Q356R & BRCA1 & rs1799950 & 72 & 12 & 0.70898 & 4 & no \\
\hline S1040N & BRCA1 & rs4986852 & 11 & 7 & 0.66416 & 3 & no \\
\hline K1690N & BRCA2 & rs56087561 & 10 & 2 & 0.536316 & 2 & no \\
\hline N3124l & BRCA2 & rs28897759 & 6 & 2 & 0.618793 & 4 & yes \\
\hline R1347G & BRCA1 & rs28897689 & 6 & 7 & 0.540272 & 2 & no \\
\hline Q2858H & BRCA2 & - & 3 & 2 & 0.539229 & 3 & novel \\
\hline V2969M & BRCA2 & rs59004709 & 2 & 2 & 0.644176 & 3 & no \\
\hline D1152N & BRCA1 & rs80357175 & 2 & 7 & 0.596261 & 2 & no \\
\hline T1011R & BRCA2 & rs 80358548 & 1 & 2 & 0.531615 & 2 & no \\
\hline E2236K & BRCA2 & rs41293503 & 1 & 2 & 0.591769 & 2 & no \\
\hline S2326C & BRCA2 & - & 1 & 2 & 0.599866 & 2 & novel \\
\hline $12627 \mathrm{~F}$ & BRCA2 & rs80359014 & 1 & 2 & 0.663608 & 4 & yes \\
\hline L2865F & BRCA2 & - & 1 & 2 & 0.593408 & 3 & novel \\
\hline V1791L & BRCA1 & rs145758886 & 1 & 11 & 0.574356 & 3 & no \\
\hline Y1703C & BRCA1 & - & 1 & 14 & 0.622922 & 4 & novel \\
\hline A1669S & BRCA1 & rs 80357087 & 1 & 13 & 0.540083 & 3 & no \\
\hline M1628T & BRCA1 & rs4986854 & 1 & 13 & 0.526967 & 1 & no \\
\hline E1346K & BRCA1 & rs 80357407 & 1 & 7 & 0.540139 & 1 & no \\
\hline $\mathrm{R} 866 \mathrm{H}$ & BRCA1 & rs80356911 & 1 & 7 & 0.676062 & 4 & no \\
\hline N723D & BRCA1 & rs4986845 & 1 & 7 & 0.543268 & 1 & no \\
\hline $\mathrm{N} 550 \mathrm{H}$ & BRCA1 & rs56012641 & 1 & 9 & 0.681765 & 4 & no \\
\hline F486L & BRCA1 & rs55906931 & 1 & 9 & 0.558913 & 1 & no \\
\hline Y179C & BRCA1 & rs56187033 & 1 & 20 & 0.588385 & 4 & no \\
\hline G602R & BRCA1 & - & 1 & 14 & 0.660547 & 4 & novel \\
\hline
\end{tabular}

Condel count: number of transcripts in which a given variant is deleterious; Condel max: maximal score from Condel; Condel algorithms: maximum number of algorithms (used in Condel score computation) in which a given variant is marked as deleterious.

detected in three patients each (Figure 1). Overall, sequencing of $B R C A 1 / 2$ coding exons identified additional predicted cancer risk mutations in 52 out of $512(10 \%)$ patients with familial and/or an early onset breast/ovarian cancer that were missed using our standard genotyping procedure. The incidence of new pathogenic mutations was higher in women with familial breast and/or ovarian cancer [26 mutations (17.9\%) in 145 patients] than those representing only familial breast cancer $[26(7.1 \%)$ in 367 patients]. These mutations could be used for estimation of cancer risk in index patients and for cascade screening.

The pathogenicity of nonsense and frameshift mutations was relatively easily established; however, the functional consequences of several missense, intronic, and small inframe insertion/deletions were not as evident, and reaching an adequate conclusion and providing advice regarding their significance were challenging tasks. These unclassified VUSs are not communicated to the patient or to non-genetic professionals to avoid increasing healthcare utilization and costs associated with unnecessary physician visits and imaging tests [38-41]. Therefore, it is urgent to develop functional studies to evaluate the pathogenicity of a suspected variant and determine its involvement in breast and/or ovarian cancer development. New genome editing techniques enable the assessment of genetic variants, including those of uncertain significance, in a native chromosomal context [42]. For example, in a proof of concept study, known mutations and artificial variants in exon18 BRCA1 were introduced using the CRISPR/Cas9 technique, resulting in traceable changes in BRCA1 mRNA splicing and abundance [43]. Such an approach, when scaled up, allows the evaluation of the combination effects of variants on splicing and transcript levels in any gene.

The overall mutation prevalence in the combined results of previous targeted genotyping studies [7] and the present amplicon sequencing was $>30 \%$ in a group of patients with early onset breast/ovarian cancer. However, the negative results of genetic testing may not be universally informative for assessing cancer risk and do not rule out hereditary predisposition. The lack of detection of pathogenic mutations in families affected by an increased risk of breast/ ovarian cancer may result not only from misinterpretation 
Table 4 Pathogenic indels in BIC, ClinVar, or SIFT indel prediction

\begin{tabular}{|c|c|c|c|c|c|c|}
\hline Variant & rs & Type & $\mathrm{BIC}$ & ClinVar & SIFT & Count \\
\hline & BRCA1 & & & & & \\
\hline c.4516delG & rs273900736 & $F$ & pathogenic & & not-tested & 4 \\
\hline c.5137delG & rs80357997 & $\mathrm{F}$ & pathogenic & pathogenic & not-tested & 1 \\
\hline c.1695insG & rs273897664 & $F$ & pathogenic & & not-tested & 1 \\
\hline c.340delTC & rs80357881 & F & pathogenic & & not-tested & 1 \\
\hline c.5285insG & rs80357886 & $F$ & pathogenic & & not-tested & 1 \\
\hline c.4597delGA & & F & & & pathogenic & 1 \\
\hline c.5232del7ins 12 & & F & & & pathogenic & 1 \\
\hline c.403delA & & F & & & pathogenic & 1 \\
\hline \multirow[t]{2}{*}{ c.403delAAGA } & & $\mathrm{F}$ & & & pathogenic & 1 \\
\hline & BRCA2 & & & & & \\
\hline c.7249delCA & & F & & & pathogenic & 3 \\
\hline c. $475+4$ delT & rs276174848 & IVS & unknown & likely pathogenic & not-tested & 1 \\
\hline c.696delT & rs80359630 & $\mathrm{F}$ & pathogenic & & not-tested & 1 \\
\hline c.2808delACAA & rs80359352 & $F$ & pathogenic & & not-tested & 1 \\
\hline c.1318delCTTA & & $\mathrm{F}$ & & & pathogenic & 1 \\
\hline c.8840delA & & $F$ & & & pathogenic & 1 \\
\hline c.8942delA & & F & & & pathogenic & 1 \\
\hline c.9375delC & & $\mathrm{F}$ & & & pathogenic & 1 \\
\hline
\end{tabular}

IVS intronic variant near splice site, $F$ frameshift variant.

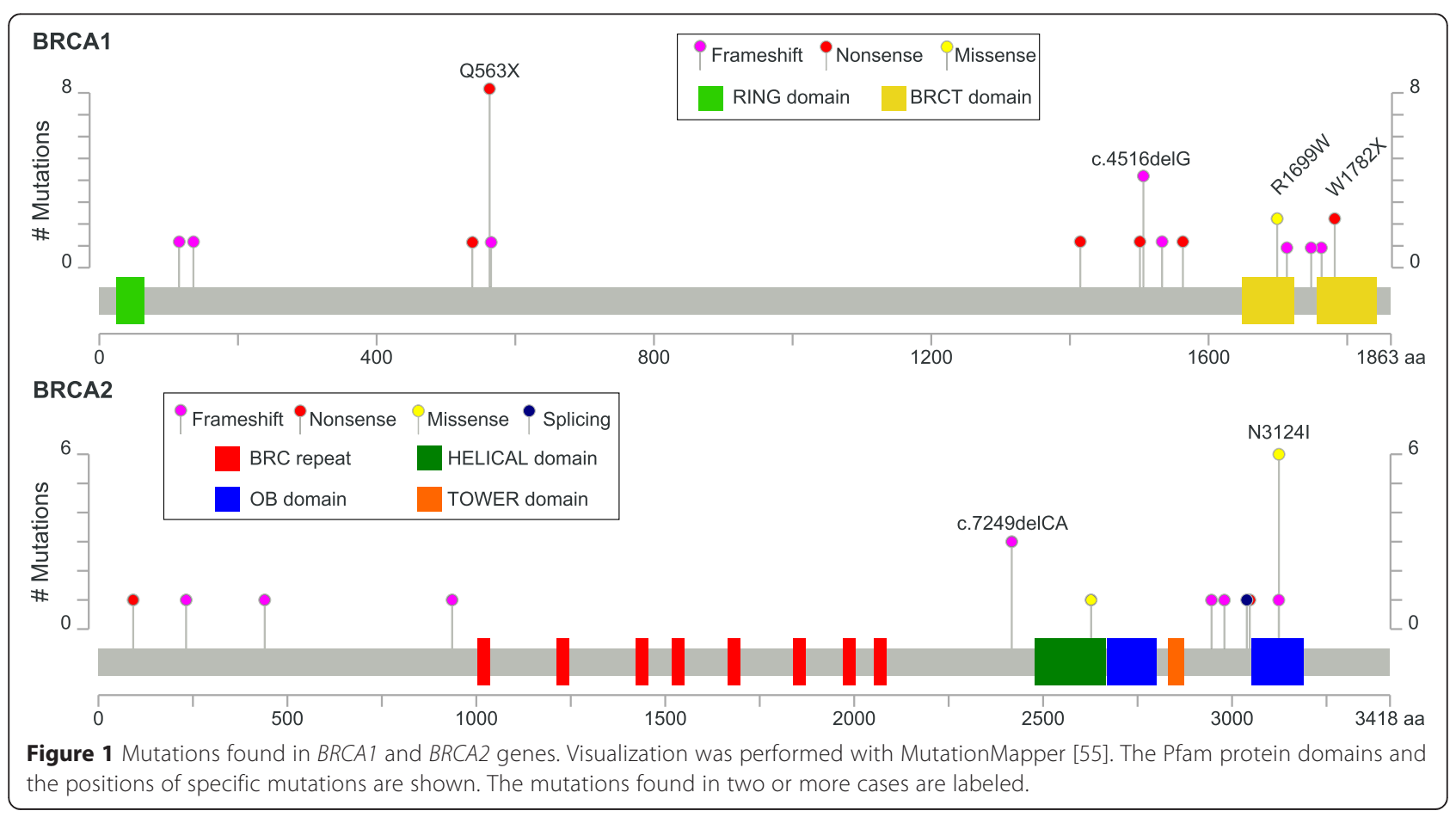


Table 5 Example of multilevel genetic testing workflow that led to identification of BRCA1 C1501X mutation in multiple family members during conducting this study

\begin{tabular}{|c|c|c|c|}
\hline Testing round & Testing workflow & Methods & Results \\
\hline | & $\begin{array}{l}\text { Targeted screening } \\
\text { using PCR-based } \\
\text { technology }\end{array}$ & $\begin{array}{l}\text { TaqMan } \\
\text { SNP } \\
\text { Genotyping } \\
\text { Assays }\end{array}$ & $\begin{array}{l}\text { Negative for } \\
\text { selected variants* }\end{array}$ \\
\hline$\|$ & $\begin{array}{l}\text { Searching for new } \\
\text { pathogenic variants } \\
\text { using NGS }\end{array}$ & $\begin{array}{l}\text { NGS of } \\
\text { BRCA1/ } \\
\text { BRCA2 } \\
\text { exons }\end{array}$ & $\begin{array}{l}\text { Pathogenic variant } \\
\text { (C1501X) found }\end{array}$ \\
\hline III & $\begin{array}{l}\text { Validation and } \\
\text { cascade screening }\end{array}$ & $\begin{array}{l}\text { Sanger } \\
\text { direct } \\
\text { sequencing }\end{array}$ & $\begin{array}{l}\text { Validation in the } \\
\text { index patient and } \\
\text { mutation found in } \\
\text { seven out of eight } \\
\text { studied relatives \# }\end{array}$ \\
\hline
\end{tabular}

*Eleven BRCA1 mutations (c.66_67delAG, C61R, c.3700_3704del5, c.3756delGTCT, c.3777delT, c.4035delA, c.4041delAG, c.4065delTCAA, c.5263delC, R1738E and R1751X) and nine BRCA2 mutations (E394X, c.5239insT, c.5946delT, c.5964delAT, c.6447delTA, c.7910del5, c.8924delT, R3128X and c.9402deIT) [7,30].

\#family tree is depicted in Additional file 2.

of determined variants [44], but also from incorrect selection of index women and/or the genetic analytical range [1]. Additionally, for some populations the large rearrangements in BRCA1/2 genes have been described in a significant proportion of families with strong breast/ovarian cancer history $[45,46]$. However, in Poland such testing is not routinely performed since no founder mutations were described [47]. In addition, amplicon sequencing with PGM platform does not seem to be a method ready for searching of $B R C A 1 / 2$ large rearrangements in genetic counseling $[11,48]$.

The presence of germline mutations in cancer susceptibility genes other than $B R C A 1 / 2$ may also lead to the development of hereditary breast/ovarian cancers. An NGS 25-gene panel revealed that the frequency of mutations in genes other than $B R C A 1 / 2$ is $4.3 \%$, of which $3.9 \%$ are in genes associated with breast/ovarian cancer [49]. The $4.3 \%$ frequency of germline mutations in additional cancer susceptibility genes was also detected through analyses of breast cancers in The Cancer Genome Atlas Project [50]. However, with the exception of PALB2, most of these predisposition genes do not reach the same degree of significance as $B R C A 1$ predisposition [51], and in contrast to $B R C A 1 / 2$ mutations, neither age at breast cancer diagnosis nor family history of ovarian or young breast cancer are predictive factors for other mutations [49].

\section{Conclusions}

NGS testing might be routine for many genes, including $B R C A 1$ and BRCA2. However, the cost of complete genetic testing is significantly higher than that of conventional PCR-based genetic testing; the internal costs in our institution, excluding personnel and overhead, are \$120 vs. \$20 [7]. Therefore, introducing the NGS to routine genotyping of BRCA (and other genes) in clinical practice would need additional financial support from public healthcare systems. Instead, targeted screening for specific BRCA1 mutations performed at the reasonable costs can be offered to all breast or ovarian cancer patients in the Polish population, regardless of the family history or the age of disease onset [7,52-54]. Since PCR-based cost-efficient testing for $B R C A 1$ and BRCA2 can detect mainly pre-selected mutations, the more recurrent mutations included in the screening, the greater efficiency of the testing.

In the present study, we identified two mutations, namely, Q563X (BRCA1) and N3124I (BRCA2), showing a strong founder effect in the Polish patients with a hereditary risk of ovarian/breast cancer. These mutations should be included in the set of mutations analyzed in PCR-based targeted screening programs in Poland. Sanger direct sequencing should be used to confirm the presence of a pathogenic mutation detected by PCR- or NGS-based genotyping, and it is the method of choice for cascade screening of relatives of the index patient. Finally, NGSbased testing of all coding exons in both genes may be reserved for patients with breast or ovarian cancer that is more likely related to $B R C A$. These considerations for the comprehensive assessment of pathogenic mutations in breast and ovarian cancers are exemplified for BRCA1 C1501X mutation found in multiple family members during conducting this study (Table 5 and Additional file 2). However, further studies are required to determine which clinical features may better define a feasible link between breast/ovarian cancer and BRCA1/2 mutations.

\section{Additional files}

Additional file 1: Contains Table S1 and Table S2 with a list of single nucleotide variants and indels, respectively, found in BRCA1/2 genes. Additional file 2: Family tree harboring C1501X mutation in BRCA1. BRC; breast cancer, RRM; risk reduction mastectomy, RRA; risk reduction adnexectomy; DLBCL; diffuse large B-cell lymphoma.

\section{Abbreviations}

BRCA 1: Breast cancer 1, early onset; BRCA2: Breast cancer 2, early onset; NGS: Next generation sequencing; SNV: Single nucleotide variants; BIC: Breast Information Core; VUS: Variant of uncertain significance.

\section{Competing interests}

The authors declare that they have no competing interests.

\section{Authors' contributions}

JO, MM conceived the study, participated in the research design, analyzed and interpreted the data, and drafted the manuscript. AK, AB, AP performed the experiments, analyzed the data. MK analyzed the data and contributed to manuscript drafting. DN provided samples and compiled clinical data. All authors have read and approve of the final version of the manuscript.

\section{Acknowledgements}

This work was supported by grant from Ministry of Science and Higher Education within the frames of the luventus Plus programme (IP2012 041172, supporting MM). 


\section{Author details}

${ }^{1}$ Department of Genetics, Maria Sklodowska-Curie Memorial Cancer Center and Institute of Oncology, 02-781 Warsaw, Poland. ${ }^{2}$ Department of Gastroenterology, Hepatology and Clinical Oncology, Medical Center for Postgraduate Education, 01-813 Warsaw, Poland.

Received: 14 February 2015 Accepted: 23 April 2015 Published online: 07 May 2015

\section{References}

1. Oosterwijk JC, de Vries J, Mourits MJ, de Bock GH. Genetic testing and familial implications in breast-ovarian cancer families. Maturitas. 2014;78:252-7.

2. Szabo C, Masiello A, Ryan JF, Brody LC. The breast cancer information core: database design, structure, and scope. Hum Mutat. 2000;16:123-31.

3. Brozek I, Ratajska M, Piatkowska M, Kluska A, Balabas A, Dabrowska M, et al. Limited significance of family history for presence of BRCA1 gene mutation in Polish breast and ovarian cancer cases. Fam Cancer. 2012;11:351-4.

4. Turner NC, Reis-Filho JS. Basal-like breast cancer and the BRCA1 phenotype. Oncogene. 2006:25:5846-53.

5. Mavaddat N, Antoniou AC, Easton DF, Garcia-Closas M. Genetic susceptibility to breast cancer. Mol Oncol. 2010;4:174-91.

6. Peto J, Collins N, Barfoot R, Seal S, Warren W, Rahman N, et al. Prevalence of BRCA1 and BRCA2 gene mutations in patients with early-onset breast cancer. J Natl Cancer Inst. 1999;91:943-9.

7. Gaj P, Kluska A, Nowakowska D, Bałabas A, Piątkowska M, Dabrowska M, et al. High frequency of BRCA1 founder mutations in Polish women with nonfamilial breast cancer. Fam Cancer. 2012;11:623-8.

8. Armstrong J, Toscano M, Kotchko N, Friedman S, Schwartz MD, Virgo KS, et al. American BRCA Outcomes and Utilization of Testing (ABOUT) Study: a pragmatic research model that incorporates personalized medicine/patientcentered outcomes in a real world setting. J Genet Couns. 2015;24:18-28.

9. Rabbani B, Tekin M, Mahdieh N. The promise of whole-exome sequencing in medical genetics. J Hum Genet. 2014;59:5-15.

10. Couch FJ, Nathanson KL, Offit K. Two decades after BRCA: setting paradigms in personalized cancer care and prevention. Science. 2014;343:1466-70.

11. Feliubadaló L, Lopez-Doriga A, Castellsagué E, del Valle J, Menéndez M, Tornero $E$, et al. Next-generation sequencing meets genetic diagnostics: development of a comprehensive workflow for the analysis of BRCA1 and BRCA2 genes. Eur J Hum Genet. 2013:21:864-70.

12. Chan M, Ji SM, Yeo ZX, Gan L, Yap E, Yap YS, et al. Development of a nextgeneration sequencing method for BRCA mutation screening: a comparison between a high-throughput and a benchtop platform. J Mol Diagn. 2012;14:602-12.

13. Wen H, Kim YC, Snyder C, Xiao F, Fleissner EA, Becirovic D, et al. Familyspecific, novel, deleterious germline variants provide a rich resource to identify genetic predispositions for BRCAx familial breast cancer. BMC Cancer. 2014;14:470

14. Ruiz A, Llort G, Yagüe C, Baena N, Viñas M, Torra M, et al. Genetic testing in hereditary breast and ovarian cancer using massive parallel sequencing. Biomed Res Int. 2014;2014:542541

15. Costa JL, Sousa S, Justino A, Kay T, Fernandes S, Cirnes L, et al. Nonoptical massive parallel DNA sequencing of BRCA1 and BRCA2 genes in a diagnostic setting. Hum Mutat. 2013;34:629-35.

16. Yeo ZX, Wong JCL, Rozen SG, Lee ASG. Evaluation and optimisation of indel detection workflows for ion torrent sequencing of the BRCA1 and BRCA2 genes. BMC Genomics. 2014;15:516.

17. McLaren W, Pritchard B, Rios D, Chen Y, Flicek P, Cunningham F. Deriving the consequences of genomic variants with the Ensembl API and SNP Effect Predictor. Bioinformatics. 2010;26:2069-70.

18. González-Pérez A, López-Bigas N. Improving the assessment of the outcome of nonsynonymous SNVs with a consensus deleteriousness score. Condel Am J Hum Genet. 2011;88:440-9.

19. Hu J, Ng PC. SIFT Indel: predictions for the functional effects of amino acid insertions/deletions in proteins. PLoS One. 2013;8:e77940.

20. Capanu M, Concannon P, Haile RW, Bernstein L, Malone KE, Lynch CF, et al. Assessment of rare BRCA1 and BRCA2 variants of unknown significance using hierarchical modeling. Genet Epidemiol. 2011;35:389-97.

21. Abkevich V, Zharkikh A, Deffenbaugh AM, Frank D, Chen Y, Shattuck D, et al. Analysis of missense variation in human BRCA1 in the context of interspecific sequence variation. J Med Genet. 2004:41:492-507.
22. Borg A, Haile RW, Malone KE, Capanu M, Diep A, Törngren T, et al. Characterization of BRCA1 and BRCA2 deleterious mutations and variants of unknown clinical significance in unilateral and bilateral breast cancer: the WECARE study. Hum Mutat. 2010;31:E1200-40.

23. Lindor NM, Guidugli L, Wang X, Vallée MP, Monteiro ANA, Tavtigian S, et al. A review of a multifactorial probability-based model for classification of BRCA1 and BRCA2 variants of uncertain significance (VUS). Hum Mutat. 2012;33:8-21.

24. Easton DF, Deffenbaugh AM, Pruss D, Frye C, Wenstrup RJ, Allen-Brady K, et al. A systematic genetic assessment of 1,433 sequence variants of unknown clinical significance in the BRCA1 and BRCA2 breast cancer-predisposition genes. Am J Hum Genet. 2007;81:873-83.

25. Spurdle AB, Lakhani SR, Healey S, Parry S, Da Silva LM, Brinkworth R, et al. Clinical classification of BRCA1 and BRCA2 DNA sequence variants: the value of cytokeratin profiles and evolutionary analysis-a report from the kConFab Investigators. J Clin Oncol. 2008;26:1657-63.

26. Karchin R, Agarwal M, Sali A, Couch F, Beattie MS. Classifying variants of undetermined significance in BRCA2 with protein likelihood ratios. Cancer Inform. 2008;6:203-16.

27. Lee T-C, Lee ASG, Li K-B. Incorporating the amino acid properties to predict the significance of missense mutations. Amino Acids. 2008;35:615-26.

28. Osorio A, Milne RL, Honrado E, Barroso A, Diez O, Salazar R, et al. Classification of missense variants of unknown significance in BRCA1 based on clinical and tumor information. Hum Mutat. 2007:28:477-85.

29. Farrugia DJ, Agarwal MK, Pankratz VS, Deffenbaugh AM, Pruss D, Frye C, et al. Functional assays for classification of BRCA2 variants of uncertain significance. Cancer Res. 2008;68:3523-31.

30. Balabas A, Skasko E, Nowakowska D, Niwinska A, Blecharz P. Novel germline mutations in BRCA2 gene among breast and breast-ovarian cancer families from Poland. Fam Cancer. 2010;9:267-74.

31. McCarthy AM, Armstrong K. The role of testing for BRCA1 and BRCA2 mutations in cancer prevention. JAMA Intern Med. 2014;174:1023-4.

32. Górski B, Jakubowska A, Huzarski T, Byrski T, Gronwald J, Grzybowska E, et al. A high proportion of founder BRCA1 mutations in Polish breast cancer families. Int J Cancer. 2004;110:683-6.

33. Thomassen M, Hansen TVO, Borg A, Lianee HT, Wikman F, Pedersen IS, et al. BRCA1 and BRCA2 mutations in Danish families with hereditary breast and/ or ovarian cancer. Acta Oncol. 2008;47:772-7.

34. Claes K, Poppe B, Coene I, Paepe AD, Messiaen L. BRCA1 and BRCA2 germline mutation spectrum and frequencies in Belgian breast/ovarian cancer families. Br J Cancer. 2004:90:1244-51.

35. Borg A, Dørum A, Heimdal K, Maehle L, Hovig E, Møller P. BRCA1 1675delA and 1135ins A account for one third of Norwegian familial breast-ovarian cancer and are associated with later disease onset than less frequent mutations. Dis Markers. 1999;15:79-84.

36. Håkansson S, Johannsson O, Johansson U, Sellberg G, Loman N, Gerdes AM, et al. Moderate frequency of BRCA1 and BRCA2 germ-line mutations in Scandinavian familial breast cancer. Am J Hum Genet. 1997:60:1068-78.

37. Antoniou AC, Hardy R, Walker L, Evans DG, Shenton A, Eeles R, et al. Predicting the likelihood of carrying a BRCA1 or BRCA2 mutation: validation of BOADICEA, BRCAPRO, IBIS, Myriad and the Manchester scoring system using data from UK genetics clinics. J Med Genet. 2008:45:425-31.

38. Domchek SM, Bradbury A, Garber JE, Offit K, Robson ME. Multiplex genetic testing for cancer susceptibility: out on the high wire without a net? J Clin Oncol. 2013:31:1267-70.

39. Hanoch Y, Miron-Shatz T, Rolison JJ, Ozanne E. Understanding of BRCA1/2 genetic tests results: the importance of objective and subjective numeracy. Psychooncology. 2014;23:1142-8.

40. Plon SE, Eccles DM, Easton D, Foulkes WD, Genuardi M, Greenblatt MS, et al. Sequence variant classification and reporting: recommendations for improving the interpretation of cancer susceptibility genetic test results. Hum Mutat. 2008;29:1282-91.

41. Mohammadi L, Vreeswijk MP, Oldenburg R, van den Ouweland A, Oosterwijk JC, van der Hout AH, et al. A simple method for cosegregation analysis to evaluate the pathogenicity of unclassified variants; BRCA1 and BRCA2 as an example. BMC Cancer. 2009;9:211.

42. Shendure J. Life after genetics. Genome Med. 2014;6:86

43. Findlay GM, Boyle EA, Hause RJ, Klein JC, Shendure J. Saturation editing of genomic regions by multiplex homology-directed repair. Nature. 2014;513:120-3. 
44. Foley SB, Rios JJ, Mgbemena VE, Robinson LS, Hampel HL, Toland AE, et al. Use of whole genome sequencing for diagnosis and discovery in the cancer genetics clinic. EBioMed. 2015;2:74-81.

45. Ticha I, Kleibl Z, Stribrna J, Kotlas J, Zimovjanova M, Mateju M, et al. Screening for genomic rearrangements in BRCA1 and BRCA2 genes in Czech high-risk breast/ovarian cancer patients: high proportion of population specific alterations in BRCA1 gene. Breast Cancer Res Treat. 2010;124:337-47.

46. Sluiter MD, van Rensburg EJ. Large genomic rearrangements of the BRCA1 and BRCA2 genes: review of the literature and report of a novel BRCA mutation. Breast Cancer Res Treat. 2011;125:325-49.

47. Ratajska M, Brozek I, Senkus-Konefka E, Jassem J, Stepnowska M, Palomba G, et al. BRCA1 and BRCA2 point mutations and large rearrangements in breast and ovarian cancer families in Northern Poland. Oncol Rep. 2008;19:263-8.

48. Tarabeux J, Zeitouni B, Moncoutier V, Tenreiro H, Abidallah K, Lair S, et al. Streamlined ion torrent PGM-based diagnostics: BRCA1 and BRCA2 genes as a model. Eur J Hum Genet. 2014;22:535-41.

49. Tung N, Battelli C, Allen B, Kaldate R, Bhatnagar S, Bowles K, et al. Frequency of mutations in individuals with breast cancer referred for BRCA1 and BRCA2 testing using next-generation sequencing with a 25-gene panel. Cancer. 2015;121:25-33.

50. Cancer Genome Atlas Network. Comprehensive molecular portraits of human breast tumours. Nature. 2012;490:61-70.

51. Lynch H, Synder C, Wang SM. Considerations for comprehensive assessment of genetic predisposition in familial breast cancer. Breast J. 2015;21:67-75.

52. Cybulski C, Lubiński J, Wokołorczyk D, Kuźniak W, Kashyap A, Sopik V, et al. Mutations predisposing to breast cancer in 12 candidate genes in breast cancer patients from Poland. Clin Genet. 2014; doi: 10.1111/cge.12524.

53. Ratajska M, Krygier M, Stukan M, Kuźniacka A, Koczkowska M, Dudziak M, et al. Mutational analysis of BRCA1/2 in a group of 134 consecutive ovarian cancer patients: novel and recurrent BRCA1/2 alterations detected by next generation sequencing. J Appl Genet. 2015;56:193-8.

54. Wong-Brown MW, Meldrum CJ, Carpenter JE, Clarke CL, Narod SA, Jakubowska $A$, et al. Prevalence of BRCA1 and BRCA2 germline mutations in patients with triple-negative breast cancer. Breast Cancer Res Treat. 2015;150:71-80.

55. Gao J, Aksoy BA, Dogrusoz U, Dresdner G, Gross B, Sumer SO, et al. Integrative analysis of complex cancer genomics and clinical profiles using the cBioPortal. Sci Signal. 2013;6:pl1

\section{Submit your next manuscript to BioMed Central and take full advantage of:}

- Convenient online submission

- Thorough peer review

- No space constraints or color figure charges

- Immediate publication on acceptance

- Inclusion in PubMed, CAS, Scopus and Google Scholar

- Research which is freely available for redistribution

Submit your manuscript at www.biomedcentral.com/submit 